\title{
Kirchliche Jugendpublizistik in Osterreich
}

\author{
von Heinz Pürer
}

Das kommunikative Engagement der Kirchen sowohl im originärpublizistischen Bereich wie auch in jenem der medialen Publizistik ist unbestritten. ${ }^{1}$ In den Bereich dieser kirchlichen Kommunikationsbemühungen fällt auch die kirchliche Jugendpublizistik. Sie stellt ihrerseits wiederum nur einen Teil kirchlicher Jugendarbeit überhaupt dar, die aus vorwiegend pädagogischen und pastoralen Erkenntnissen zunächst „zu einem besonderen Betätigungsfeld der Seelsorge und christlichen Erziehung $^{\text {“2 }}$ wurde, primär religiöse, pädagogische und soziale Ziele verfolgte und kirchlichen Intentionen der Lebenshilfe und -bewältigung diente. ${ }^{3}$ Mittlerweile hat sich kirchliche Jugendarbeit auf alle soziokulturellen und nicht selten soziopolitischen Bereiche ausgedehnt. Sie wird in Osterreich - freilich analog zum Verhältnis der Realpräsenz - von der katholischen und evangelischen Kirche in gleicher Weise geleistet.

Das Ziel der vorliegenden Untersuchung ist es, das Kommunikationsgefüge der kirchlichen (Presse-) Jugendpublizistik Österreichs aufzuzeigen. Publizistikwissenschaftlichen Fragestellungen gemäß wurden dazu alle am Prozeß kirchlicher Jugendpublizistik beteiligten Faktoren abgefragt. ${ }^{4}$ Die Arbeit umfaßt eine mündliche Befragung der Redakteure (= Kommunikatorposition) der national verbreiteten kirchlichen Jugendzeitschriften nach Selbstverständnis, Motiv und Intention ihrer Tätigkeit; eine Strukturuntersuchung ${ }^{5}$ der national verbreiteten kirchlichen Jugendzeitschriften im Hinblick auf das verlegerische und äußere (Position: Medium-formal) sowie spezifisch inhaltliche Gefüge (Position: Aussage/Inhalt), um eine Typologie der Zeitschriften zu erstellen und bestimmte Merkmale ihrer Inhalte abzufragen; weiters eine schriftliche Befragung von Lesern (Position: Rezipient) national verbreiteter Kinderzeitschriften im Hinblick auf Lesemotivation, Interessenstreuung und Rezeption spezifisch religiöser Inhalte. ${ }^{8}$

\section{Theoretische Überlegungen}

Der folgenden Untersuchung kirchlicher Jugendpublizistik, die unser Materialobjekt abgibt, das wir unter der Formalperspektive publizistischer Formalkategorien betrachten, sind - hier stark gekürzt wiedergegeben - theoretische Úberlegungen zugrundegelegt. Seit ihrem verhältnismäßig kurzen Bestehen hat die Wissenschaft von der Publizistik einige theoretische Ansätze entwickelt. Unter Theorie verstehen wir hier systematisch aufgebaute und geordnete Erkenntnisse bzw. unter allgemeine Gesetze subsumierte Einzelerkenntnisse. Diesem Verständnis von Theorie liegt ein Ordnungsprozeß zugrunde, der die Regelung der Beziehungen von Gegenständen bzw. Faktoren untereinander beinhaltet. In unserem Falle sind diese Gegenstände oder Faktoren die Kommunikatoren, die Aussagen (an Medien gebunden) und die Rezipienten religiöser oder kirchlicher Publizistik. Die allgemeinen Gesetze, unter

Dr. phil. Heinz Pürer ist Hochschulassistent am Institut für Publizistik und Kommunikationstheorie der Universität Salzburg. Bei dem vorliegenden Aufsatz handelt es sich um die stark gekürzte Fassung von Ergebnissen seiner Dissertation „Kirchliche Jugendpublizistik in Osterreich. Eine Analyse des Kommunikationsgefüges kirchlicher Publizistik am Beispiel ausgewählter konfessioneller Kinder- und Jugendzeitschriften", Salzburg 1973. 
die unsere Erkenntnisse eingeordnet sind, beziehen sich auf theoretische Überlegungen eines normativen Verständnisses von Publizistik.

Ein solcher Ansatz mag dem Publizistikwissenschaftler von heute als überholt erscheinen, zumal die Theorienbildung innerhalb des Faches ja doch weiter fortgeschritten ist und ihr Interesse vor allem auf kritisch-theoretische und insbesondere systemtheoretische Ansätze bezieht. ${ }^{7}$ Dem hier vertretenen normativen Verständnis von Publizistik liegt die Hypothese zugrunde, daß kirchliche Publizistik im allgemeinen sich nicht orientiert an ihrem Partner im publizistischen Prozeß, am Rezipienten (bzw. am Gläubigen), sondern auf ihn einzuwirken sucht, um ihn in seinem Tun und Handeln zu bestimmen. ${ }^{8}$ Dieser Denkansatz impliziert einen Kommunikator, dem „möglichst umfassende Handlungsregeln auf den Weg ${ }^{\text {"g }}$ mitgegeben werden, „die in ihren werthaltigen Kategorien die Wertungen der Lehrenden ${ }^{* 10}$ widerspiegeln. Das Publikum wird dabei "mitgedacht als zu führende und möglichst zu belehrende [...] Empfängerschaft".11 Der Kommunikationsprozeß wird dadurch - bewußt oder unbewußt - in ein monologisches, vertikal verlaufendes Verständigungsgefüge umfunktioniert ${ }^{12}$ und läßt durch das von oben nach unten verlaufende Kommunikationsgefüge den kirchlichen Kommunikator als "Anweisungspublizisten "13 erscheinen. Die apriorisch für wahr gehaltenen (oder für wahr zu haltenden) Kommunikationsinhalte religiöser Uberzeugung mit öffentlicher Intention erweisen sich zudem letztlich als weltanschaulich-ideologisch-religiös verwurzelt. $^{14}$ Ihre normativ-zielgerichteten Intentionen liegen in ihrem Absolutheitsanspruch, ihre Basis teils in den „Schriften“ (Evangelien), teils in der Tradition (Dogmen, Kirchengesetze usw.). ${ }^{15}$ Innerhalb der kirchlichen, von uns als normativ betrachteten Publizistik wird die Aussage als Agens, der Rezipient als Beeinflußter ${ }^{16}$, die Summe der Rezipienten gedacht als „zu führende und möglichst auch zu belehrende [...] Empfängerschaft" ${ }^{17}$

Eine sich derart präsentierende "Kommunikationsverfassung “ der Kirchen läßt das Faktum außer acht, daß die publizistische, also öffentliche und öffentlichkeitsansprucherhebende religiöse oder - durch die Kirchen institutionalisiert - kirchliche Aussage jedoch nicht einfach eine Summe von gleichgesinnten Individuen trifft, sondern, je nach Konstellation, Situationsmasse oder disperses Publikum. ${ }^{18}$ So etwa treffen für die originärpublizistische Form der, gottesdienstlichen Predigt' im klar umgrenzten Sakralraum mit möglicherweise durch die Taufe limitiertem Hörerkreis andere Gesetze zu als für die über technische (Massen-)Medien verbreiteten publizistischen Aussagen religiösen oder kirchlichen Inhalts: diese erfolgen indirekt und einseitig sowie unter anderen zeit-räumlichen Dispositionen; der potentielle Rezipientenkreis ist je nach eingesetztem Medium ungleich größer sowie in seiner geistigen und ideellen Struktur wesentlich weniger homogen.

Seit es Kirchen gibt, haben sie kirchenspezifische Kommunikationssysteme zu entwickeln und zu gestalten versucht und sich auch der technischen Massenmedien bedient, um mit ihren Anliegen in Offentlichkeit und Gesellschaft zu dringen. Wie erscheint nun dem Betrachter das Kommunikationsgefüge eines kleinen Teilbereiches konfessioneller Kommunikationsbemühungen, nämlich jenes der national verbreiteten katholischen und evangelischen (Presse-) Jugendpublizistik in Osterreich?

\section{Empirischer Teil}

Wie bereits eingangs erwähnt, werden in der vorliegenden Studie die Kommunikatoren, Medien, die an die Medien gebundenen Inhalte sowie Rezipienten der national, 
also im gesamten Bundesgebiet Osterreichs verbreiteten, von Organisationen der katholischen und evangelischen Kirche( $\mathrm{n}$ ) herausgegebenen und verantworteten kirchlichen Kinder- und Jugendzeitschriften ${ }^{18}$ untersucht, um zu Aussagen über das Kommunikationsgefüge kirchlicher (Presse-)Jugendpublizistik in Osterreich zu gelangen. Die dabei angewendeten Methoden sowie gewonnenen Erkenntnisse und daraus abzuleitenden publizistik- bzw. kommunikationswissenschaftlichen Schlußfolgerungen werden in den nachfolgenden Kapiteln, gegliedert in Analogie zum publizistischen Prozeß, dargestellt.

\section{Die Redakteure kirchlicher Jugendzeitschriften (Kommunikatorposition)}

An den Beginn der Untersuchung ist eine Kommunikatorstudie gestellt, in der alle Redakteure der zwölf national verbreiteten kirchlichen Jugendzeitschriften über die Bereiche ihrer Tätigkeit bei den von ihnen gemachten Medien innerhalb der Institution Kirche sowie über die daraus sich ergebenden Bedingungen und Konsequenzen für die Arbeit in den Zeitschriften befragt werden sollten. Diese 25 Personen umfassende Gruppe ist zugleich identisch mit den für die Inhalte der Zeitschriften sowie ihren Auftraggebern, also verschiedenen kirchlichen Organisationen gegenüber, verantwortlichen Redakteuren. So gesehen entspricht diese Gesamtheit der Befragten der Grundgesamtheit aller in den von uns zur Beurteilung ausgewählten Pressemedien hauptamtlich oder nebenberuflich tätigen Redakteuren, und im Hinblick auf die von uns zu untersuchenden Zeitschriften einer Totalerhebung. Die für die RedakteurUmfrage notwendigen Interviews wurden mittels eines halbstrukturierten Fragebogens mit teils offenen, teils geschlossenen Fragen mündlich in Form von Leitfadengesprächen in Wien, Salzburg, Innsbruck, Graz und Klagenfurt durchgeführt. Trotz mehrmaliger Versuche konnten vier der 25 zu befragenden Personen nicht erreicht werden. Die Ausfallquote beträgt somit 16 Prozent, die Grundgesamtheit der Befragten sind 21 Redakteure (= 100 Prozent).

Dieser in den Bereich der Kommunikatorforschung fallende Teil der Studie, der sich in erster Linie mit der Figur des Kommunikators als einem Grundfaktor des Prozesses öffentlicher Kommunikation, in unserem Falle öffentlicher kirchlicher Kommunikation mit umgrenzbarer Zielgruppe (nämlich Kindern und Jugendlichen), beschäftigt, versucht, Fragen der Personalstruktur der Gesamtheit der Redakteure, Motive und Intentionen ihrer Tätigkeit, redaktionelle Abhängigkeiten und journalistische Leistungen (Bestimmung und Gestaltung des Inhalts) sowie das Leser-Bild der Kommunikatoren abzuklären. Die Umfrage hat die nachfolgend kurz zusammengefaßten, wesentlichen Ergebnisse gebracht:

- Die Redakteure kirchlicher Jugendpublizistik rekrutieren sich zu über 80 Prozent aus in den Kirchen bzw. kirchlichen Organisationen tätigen Laien durchaus unterschiedlichen Bildungsstandes und Alters, wobei jedoch Personen im Alter zwischen 20 und 30 Jahren in der Mehrzahl vertreten sind. Die Personalstruktur der Redakteure widerlegt (für die kirchliche Pressejugendpublizistik Österreichs) die Ansicht, wonadh kirchliche Kommunikatorpositionen vornehmlich auf Kleriker- und Theologenkreise beschränkt würden. ${ }^{20}$ Kirchliche Jugendpressearbeit wird haupt- und nebenberuflich ausgeübt, vornehmlich jedoch im Zusammenhang mit anderen Tätigkeiten in der kirchlichen Jugendarbeit, so daß aufgrund der ständigen Begegnung mit Kindern und Jugendlichen zumindest zum Teil eine Form bzw. Möglichkeit der Rückkoppelung besteht. Die Redakteure sind Religion und Glauben gegenüber sehr positiv eingestellt und erweisen sich - verbal - anderen Konfessionen und deren Institutionen gegenüber als aufgeschlossen und tolerant. 
- Die von uns befragten Redakteure verfolgen in erster Linie religiöse, caritative und soziale Ziele. Durch die (vermeintliche, wie noch zu zeigen sein wird) schwerpunktmäßige Berichterstattung über Religion, Caritatives und Soziales sollen Handlungsorientierung und -anweisung, Schaffung von Problembewußtsein und Konfliktlösungsmodelle in verschiedenen Lebensbereichen angeboten werden. Außerschulische Bildungsarbeit und das Anbieten von Unterhaltung sind eher nachrangig eingestufte publizistische Ziele der Kommunikatoren.

- Ein überraschend großer Anteil der Befragten, nämlich wieder über 80 Prozent, fühlt sich trotz bestehender Richtlinien, wie die Zeitschrift zu machen ist, seitens der Auftraggeber weisungsungebunden und redaktionell unabhängig. Er versteht sich als der zwar teils im Auftrag der Kirche schreibende, sich jedoch mit seiner Tätigkeit identifizierende und nach Aktualisierung (der zu vermittelnden Inhalte) suchende Jugendpublizist.

- Die Redakteure wenden sich mit ihren Medien nicht an einen ausschließlich konfessionell gebundenen Leserkreis, sondern versuchen, eine breite Zielgruppe anzusprechen. Ihre zumeist aber doch der katholischen oder den evangelischen Kirchen angehörenden Leser sehen sie als zuwenig aktiv und re-aktiv, und sie vermissen derenseits ein (engagiertes) feed back.

\section{Die Zeitschriften kirchlicher Jugendpublizistik}

Als zweite Position innerhalb des publizistischen Prozesses sind die zwölf national verbreiteten kirchlichen Jugendzeitschriften struktur- und inhaltsanalytisch bearbeitet worden. Die als besondere Form der Inhaltsanalyse für kommunikationswissenschaftliche Untersuchungen angewendete Methode der Strukturanalyse ${ }^{21}$ leistet die deskriptive und quantifizierende Erfassung der formal äußeren und redaktionell inneren (inhaltlichen) Struktur von Pressemedien ${ }^{22}$ und umfaßt hier die Typologie der sich an spezifische Zielgruppen wendenden, unserer Analyse zugrundeliegenden Zeitschriften; weiters eine Erfassung des Gesamtbestandes der Titel, Auflage, drucktechnischen Gestaltung und wirtschaftlichen Nutzung durch Anzeigen; schließlich in der Analyse der inneren Struktur anhand eines eigens entwickelten (Inhalts-)Kategorienschemas bestimmte Merkmale ihres Inhalts.

\subsection{Typologie}

Die von Organisationen der katholischen und evangelischen Kirche(n) herausgegebenen $z$ wölf national verbreiteten kirchlichen Jugendzeitschriften lassen sich ohne Unterschied bezüglich der Konfession in drei Gruppen einteilen und ermöglichen eine Typologie in "Kinderzeitschriften ", "Jugendzeitschriften " und "Führungszeitschriften", die sich, ihren jeweiligen Zielgruppen von Kindern, Jugendlichen und in der kirchlichen Jugendarbeit tätigen Führungskräften angeglichen, in Form und Aussage durch spezifische Inhalte ausweisen. Innerhalb dieser Typologie zählen zu den Zeitschriftengruppen die folgenden Titel:

Kinderzeitschriften: „Die Arche" (ev., Auflage 5.700); "Gotteskinder" (kath., A: 45.000); „Pfeil" (kath., A: 70.000); "Weite Welt" (kath., A: 80.000).

Jugendzeitschriften: "anstoss" mit Beilage "argumente" (ev., A: 7.000); "notizen" (kath., A: 2.000); "opal“ (kath., A: 23.000); "reflexionen“ (kath., A: 1.000). 
Führungszeitschriften: „aktion" (kath., A: 1.700); "Junge Gemeinde" (ev., A: 1.100); "Jungschar“ (kath., A: 6.000); „Unser Dienst“ (ev., A: 500).

\subsection{Die verlegerische und äußere Struktur}

Zum Zeitpunkt der Untersuchung (1971/72) erschienen in Osterreich insgesamt 12 überregional verbreitete katholische und evangelische Jugendzeitschriften ${ }^{23}$, die seitens der katholischen Kirche von einigen in den Dachverband der Arbeitsgemeinschaft Katholischer Jugend Österreichs eingegliederten Suborganisationen, auf evangelischer Seite vom Evangelischen Jugendwerk oder seinen Sektionen publiziert wurden. Thre Gesamtauflage betrug pro Ausgabe 242.900 Exemplare. Davon entfallen 200.700 Exemplare (oder 82,6 Prozent) auf die Kinderzeitschriften, 32.900 Exemplare (oder 13,6 Prozent) auf die Jugendzeitschriften und 9.300 Exemplare (oder 3,8 Prozent) auf die Führungszeitschriften.

- Mit insgesamt vier Zeitschriften - einem Drittel aller Titel - kann die evangelische Kirche im Hinblick auf die Druckauflage (pro Ausgabe) ihrer Zeitschriften mit 14.300 Exemplaren einen Anteil von 6 Prozent an der Gesamtauflage für sich buchen. Angesichts der verhältnismäßig geringen Realpräsenz der evangelischen Kirche in Osterreich, - ihr Anteil an christlich Getauften beträgt 6,5 Prozent der Gesamtbevölkerung Osterreichs ${ }^{24}$, - überrascht dieses Ergebnis weder positiv noch negativ. Von ihrer formalen, äußeren Erscheinung her sind die evangelischen Zeitschriften drucktechnisch allgemein wenig aufwendig gestaltet.

- Acht Zeitschriften werden von Organisationen der katholischen Kirche herausgebracht, die mit zwei Dritteln der Titel und einem Anteil von 228.600 Exemplaren 94 Prozent der Gesamtauflage aller zwölf untersuchten Zeitschriften darstellen. Die katholischen Zeitschriften zeichnen sich im Vergleich zu den evangelischen Zeitschriften durch eine teilweise anspruchsvolle drucktechnische Gestaltung aus, wodurch sie in der Aufmachung kommerziellen Kinder- und Jugendmagazinen angeglichen erscheinen.

- Die auflagenstärkste Gruppe der national verbreiteten, periodisch erscheinenden kirchlichen Jugendzeitschriften Osterreichs bilden mit einem Anteil von 82,6 Prozent an der Gesamtauflage (pro Ausgabe) die Kinderzeitschriften, die durchschnittlich auch am häufigsten erscheinen und in aufwendigen Mehrfarben-Druckverfahren hergestellt werden.

- Mit 13,6 Prozent Anteil an der Gesamtauflage stellen die Jugendzeitschriften innerhalb der hier untersuchten Blätter zwar den zweitgrößten, aber doch auflagemäßig kleinen Teil dar. Die vier in diese Gruppe fallenden Titel erscheinen je monatlich; ihre drucktechnische Herstellung ist unterschiedlich, im Durchschnitt jedoch eher wenig aufwendig. Obwohl gerade auf dem Gebiet der "Jugendzeitschriften" überhaupt die Kirchen auch in Ơsterreich mit einer verhältnismäßig starken Konkurrenz konfrontiert sind, lassen sich - mit Ausnahme der katholischen Zeitschrift "opal“, die mittlerweile jedoch als eigenständige publizistische Einheit eingestellt wurde, keine Bemühungen seitens der Kirchen erkennen, sich im Hinblick auf die formale Gestaltung von Jugendzeitschriften an die gegebene Marktsituation und ihre Erfordernisse anzupassen.

- Obwohl mit vier Titeln vertreten, erweisen sich die Führungszeitschriften als kleinste Zeitschriftengruppe: sie haben mit 9.300 Exemplaren einen Anteil von nur 3,8 Prozent an der Gesamtauflage pro Ausgabe. Die „Führungszeitschriften“, an- 
spruchslos aufgemacht, dienen Mitarbeitern kirchlicher Kinder- und Jugendarbeit und Führern von Jugendgruppen als Orientierungshilfe für die Gestaltung der Jugend arbeit in den Gruppenstunden sowie der Kommunikation untereinander.

- Alle kirchlichen Jugendzeitschriften erfahren - mit Ausnahme der evangelischen Zeitschrift "anstoss" ${ }^{\text {" }}$ - keine wirtschaftliche Nutzung; ihr nichtkommerzieller Charakter wird dadurch deutlich betont.

\subsection{Die redaktionelle, innere (inhaltliche) Struktur}

Für die Erarbeitung dieses Teiles der Studie ist zur Erfassung der Inhalte ein Kategorienschema erstellt worden, das zunächst $\mathrm{z}$ wischen den vier großen Inhaltsgruppen "religiöse“, „kirchlich-institutionelle“, „säkulare“ und „Unterhaltungs"-Inhalte unterschied. Diese vier Großgruppen waren in sich durch je verschiedene Untergruppierungen $^{25}$ thematisch-inhaltlicher Art spezifiziert; nach entsprechenden Vortests wurde das Kategorienschema, wo es sich als notwendig erwies, korrigiert, so daß schließlidh in der Erhebung die Artikel den Inhaltskategorien zugeordnet, die Ergebnisse auf Randlochkarten übertragen und in weiterer Folge die Auswertung vorgenommen werden konnten. ${ }^{26}$

- Innerhalb der für diese Untersuchung getroffenen Auswahl von Heften aus dem zweiten Quartal (April, Mai, Juni) des Jahres 1970 finden sich insgesamt 356 Artikel (= 100 Prozent). ${ }^{27}$ Davon finden sich - analog zu unserer Typologie - in den Heften der Kinderzeitschriften 99 Artikel (= 27,8 Prozent), in jenen der Jugendzeitschriften 156 Artikel (= 43,9 Prozent) sowie in den Führungszeitschriften 101 Artikel ( $=28,3$ Prozent).

- Auf die vier großen inhaltlichen Kategoriengruppen verteilt sich die Gesamtheit der 356 erhobenen Artikel wie folgt: 35 Aussageeinheiten, das entspricht knapp 10 Prozent, behandeln in den Bereich der religiösen Inhalte fallende Themen. $77 \mathrm{Ar}$ tikel oder 21,6 Prozent, entfallen auf die kirchlich-institutionellen Inhalte. 104 Aussageeinheiten, das sind 29,2 Prozent, können dem "Säkularen " zugeordnet werden. 115 Artikel oder 32,3 Prozent (und somit die größte Gruppe) sind Unterhaltungsartikel. Insgesamt 25 Texte können im Kategorienschema nicht untergebracht werden und sind unter "Sonstiges" eingeordnet.

Bei der Analyse der religiösen und kirchlich-institutionellen Beiträge ist in der Artikelerhebung auch der konfessionelle Bezug berücksichtigt worden. Von insgesamt 112 religiösen und kirchlich-institutionellen Artikeln (gemeinsam) beziehen sich 87 Aussageeinheiten eindeutig auf die eigene Lehre bzw. eigene Kirche, in nur zwei Artikeln wird über andere Kirchen berichtet, bei den restlichen 23 Aussageeinheiten ist kein eindeutiger institutioneller Bezug feststellbar. Der Anteil von Artikeln mit institutionellem Bezug zu anderen Kirchen ist damit unerwartet gering.

Hinsichtlich der publizistischen Form der Artikel überwiegen interpretative Artikel in allen Inhaltsgruppen eindeutig, weniger oft vertreten sind rein informative oder aber auch kommentierende Aussageeinheiten. Weiters finden sich insgesamt 12 meditative Artikel. Von dieser besinnlichen publizistischen Aussageform entfallen 9 Aussageeinheiten auf religiöse Themen. Der Meditation als Betrachtungsweise von Sachverhalten wird demnach in den kirchlichen Jugendzeitschriften nicht unbedingt viel Beachtung geschenkt. So wie bei allen Inhaltsgruppen gemeinsame Häufigkeiten interpretativer Artikel feststellbar sind, zeigt auch die Untersuchung der Länge der Artikel auffallende gemeinsame Häufigkeiten in allen Inhaltsgruppen bei Artikeln $z$ wischen einer und zwei Seiten, wie ebenso alle Inhaltsgruppen in relativ gleicher 
TABELLE 1 Verteilung der Artikel auf die einzelnen Inhaltskategorien

(Grundgesamtheit: 356 Artikel [= 100\%])

Religiöse Artikel:

davon entfallen auf

Theologie

Spiritualität

Verkündigung

Gemeinde

Leitbilder
$35=9,8$ Prozent aller Artikel

$=100$ Prozent der Inhaltsgruppe

Kirchlich-institutionelle Artikel: $\quad 77=21,6$ Prozent aller Artikel

$=100$ Prozent der Inhaltsgruppe

davon entfallen auf

Kirche allgemein $\quad 6=7,8 \%$

Hierarchie $\quad 1=1,3 \%$

Priester $\quad 4=5,2 \%$

kirchliche Gliederungen $\quad 38=49,3 \%$

Sozial-Karitatives $\quad 14=18,2 \%$

Kirchlich-Kulturelles $\quad 14=18,2 \%$

Säkulare Artikel

davon entfallen auf

Politik

Soziales

Kultur, Reise

Sport

Zeitgeschichte

Wissenschaft/Technik

Dritte Welt

Schule/Beruf/Erziehung

Sonstiges
$104=29,2$ Prozent aller Artikel

$=100$ Prozent der Inhaltsgruppe

\section{Unterhaltungsartikel}

davon entfallen auf

Film, Fernsehen

Mode

Schlager, Pop, Folk

Basteleien, Kochen

Rätsel

Freizeit allgemein

Erzählungen, Romane

$$
7=6,7 \%
$$

$10 \Rightarrow 9,6 \%$

$22=21,2 \%$

$18=17,3 \%$

$4=3,8 \%$

$10=9,6 \%$

$4=3,8 \%$

$20=19,3 \%$

$9=8,7 \%$

Sonstiges

$115=32,3$ Prozent aller Artikel

$=100$ Prozent der Inhaltsgruppe
$21=18,3 \%$

$10=8,7 \%$

$16=13,9 \%$

$15=13,0 \%$

$10=8,7 \%$

$17=14,8 \%$

$26=22,6 \%$

$25=7,04$ Prozent aller Artikel 
TABELLE 2 Verteilung der Inhaltsgruppen auf Zeitschriftentypen

\begin{tabular}{|c|c|c|c|}
\hline & $\begin{array}{l}\text { religiöse } \\
\text { Artikel }\end{array}$ & $\begin{array}{l}\text { kirchlich- } \\
\text { institut. } \\
\text { Artikel }\end{array}$ & $\begin{array}{l}\text { säkulare } \\
\text { Artikel }\end{array}$ \\
\hline "Kinderzeitschriften" & $9(25,8 \%)$ & $8(10,3 \%)$ & $20(18,2 \%)$ \\
\hline "Jugendzeitschriften" & $10(28,5 \%)$ & $21(27,3 \%)$ & $66(63,5 \%)$ \\
\hline \multirow[t]{3}{*}{ „Führungszeitschriften“ } & $16\left(45,7^{0} / 0\right)$ & $48(62,4 \%)$ & $18(17,3 \%)$ \\
\hline & $35(100 \%)$ & $77(100 \%)$ & $104(100 \%)$ \\
\hline & $\begin{array}{l}\text { Unter- } \\
\text { haltungs- } \\
\text { Artikel }\end{array}$ & ,Sonstiges & SUMME \\
\hline "Kinderzeitschriften" & $61(53,0 \% / 0)$ & $1(4,0 \%)$ & $99(=27,8 \%$ aller Artikel $)$ \\
\hline „Jugendzeitschriften “ & $37(32,2 \%)$ & $22(88,0 \%)$ & $156(=43,9 \%$ aller Artikel $)$ \\
\hline \multirow[t]{2}{*}{ „Führungszeitschriften“ } & $17(14,8 \%)$ & $2(8,0 \%)$ & $101(=28,3 \%$ aller Artikel $)$ \\
\hline & $115(100 \%)$ & $25(100 \%)$ & $356(=100 \%$ aller Artikel $)$ \\
\hline
\end{tabular}

Weise unterschiedlich lange Artikel aufweisen und nicht etwa eine Inhaltsgruppe nur aus kurzen, eine andere nur aus langen Artikeln besteht. Die Berechnung der Durchschnittslänge der Artikel bei Vernachlässigung der oberen Grenzkategorie „über zwei Seiten“ ergibt bei allen Inhaltsgruppen Werte bzw. Längen der Aussageeinheiten $\mathrm{z}$ wischen 1,2 und 1,5 Seiten.

Ebenso haben wir in die Erhebung noch die Illustrierung des Artikels mit einbezogen. Demnach sind 31 Prozent aller religiösen Artikel, 23 Prozent aller kirchlich-institutionellen Artikel, 47 Prozent aller säkularen Artikel und 68 Prozent aller Unterhaltungsartikel durch Fotos, Zeichnungen oder Karikaturen illustriert. Somit zeichnen. sich die säkularen und die Unterhaltungsartikel, und damit auch die beiden großen Inhaltsgruppen, durch einen hohen Grad an Illustrierung aus, während die weniger stark vertretenen Themengruppen auch weniger oft illustriert sind. Insgesamt 43,5 Prozent der Grundgesamtheit aller Aussageeinheiten weisen illustrative Gestaltung auf.

Eine Korrelation zwischen Artikelverteilung auf die Inhaltskategorien und Inhaltskategorienverteilung auf die Zeitschriftentypen erbringt die nachfolgend wieder kurz zusammengefaßten, wesentlichen Resultate:

Die national verbreiteten kirchlichen Jugendzeitschriften erweisen sich sowohl hinsichtlich ihrer formal-äußeren Erscheinung wie auch im Hinblick auf die quantitative Verteilung der Inhaltsgruppen als unterschiedlich strukturiert. In den einzelnen Zeitschriftentypen können Schwerpunkte der Berichterstattung eindeutig festgestellt werden..$^{28}$ Religiöse, kirchlich institutionelle, säkulare und Unterhaltungsartikel sind unterschiedlich auffindbar: In den Kinderzeitschriften überwiegen Unterhaltungsartikel eindeutig, in den Jugendzeitschriften die säkularen Artikel. In den Führungszeitschriften sind kirchlich-institutionelle Artikel überrepräsentiert vertreten. Als kleinste 
und in allen Zeitschriftentypen am geringsten vertretene Inhaltsgruppe erweisen sich die religiösen Artikel, die sich in den Führungszeitschriften am häufigsten noch finden. Die religiösen und kirchlich-institutionellen Artikel sind damit zusammen mit dem gemeinsamen Anteil von 33,7 Prozent aller erfaßten Artikel unerwartet gering positioniert.

- Der gemeinsame Anteil der säkularen und der Unterhaltungsartikel ist mit 66,3 Prozent doppelt so groß wie jener der religiösen und kirchlich-institutionellen. Dieses Resultat steht im Gegensatz zu den Aussagen über die Intentionen der Kommunikatoren. Die Berichterstattung dieser Inhaltsgruppen zeichnet sich durch ein verhältnismäßig breites Spektrum von Themen (Politik, Soziales, Kultur, Sport, Geschichte, Geographie, Wissenschaft und Technik, Dritte Welt, Schule, Beruf, Erziehung; Film, Fernsehen, Schlager, Pop- und Folkmusik, Mode, Basteleien, Freizeitgestaltung, Erzählungen usw.) aus. Die Berichterstattung versucht, die Kinder und/oder Jugendlichen in ihren Interessenbereichen anzusprechen.

- Die sozialen und caritativen Intentionen der Kommunikatoren schlagen sich in der relativ häufigen Berichterstattung über Soziales und über caritative Täțigkeiten der Kirchen tatsächlich nieder. Allerdings erschöpft sich diese Berichterstattung in Artikeln über Armen-, Alten-, Kranken-, Entwicklungs- und Missionshilfe. Sozialkritische Beiträge (Minderheiten, Gefangene, politisch Verfolgte etc.) sind nicht auffindbar.

- Gesellschaftskritische Probleme sowie Fragen der Lebenshilfe werden nur in einigen wenigen Artikeln aufgegriffen (Kategorie "Sonstiges"). Völlig vernachlässigt und in keinem einzigen Artikel behandelt erscheinen alle jene Probleme, die den jungen Menschen im Zusammenleben neben oder mit dem Partner des anderen Geschlechtes konfrontieren.

- Eine unterschiedliche Strukturierung erweist sich auch zwischen katholischen und evangelischen Zeitschriften. Sie besteht darin, daß die Berichterstattung der auflagenstarken großen katholischen Zeitschriften („Weite Welt", „Pfeil“, „opal“) in Gestaltung und Inhalt den kommerziellen Jugendmagazinen angeglichen erscheint und ihren Unterhaltungsteil stark ausbaut. Hingegen weist die Berichterstattung der evangelischen Zeitschriften mehr nationalen Bezug zu Staat und Kirche auf.

\section{Leser kirchlicher Jugendzeitschriften (Position ,Rezipient')}

Neben die Kommunikatorumfrage, Medien- und Inhaltsanalyse reiht sich die Untersuchung von Lesern kirchlicher "Kinderzeitschriften" als viertes Glied in die Analyse des Kommunikationsgefüges kirchlicher Jugendpublizistik. Befragt wurde hierzu eine Auswahl von 160 12- bis 13jährigen Schülern und Schülerinnen aus kirchlich geleiteten Allgemeinbildenden Höheren Schulen (mit Offentlichkeitsrecht) in Salzburg; in den diesen Schulen angegliederten Schülerheimen konnten zudem städtische und ländliche Leser erfaßt werden. Die in den Schulen und Klasseneinheiten forschungstechnisch leicht zu fassende Personengruppe war zugleich identisch mit den Zielgruppen einiger von uns analysierter, dem Personenkreis zugänglicher Kinderzeitwhriften ("Gotteskinder", „Pfeil" und "Weite Welt", sämtlich katholische Zeitchriften). Methodisch wurde für die Leser-Umfrage ein eigens erstellter, standardinierter Fragebogen verwendet, der in Anwesenheit des Bearbeiters der vorliegenden Studie in den Klassenräumen von den Schülern und Schülerinnen schriftlich zu bentworten war. Dies hatte u. a. den Vorteil, daß bei eventuell auftretenden Unklarheiten der Befragten beim Interviewer selbst Auskunft eingeholt und außerdem auf 
ein möglichst eigenständiges Ausfüllen des Fragebogens geachtet werden konnte. Potentielle Fehlerquellen sollten dadurch nach Möglichkeit ausgeschaltet werden. Durch das Abfragen von Bekanntheitsgrad und Leseintensität der kirchlichen Jugendzeitschriften im Vergleich zu den kommerziellen Jugendmagazinen, von Interessenstreuung, Themengefälligkeit und Themenwünschen sowie von Rezeption und Beurteilung der religiösen Inhalte der Zeitschriften sollte versucht werden, festzustellen, inwieweit die kirchlichen Jugendzeitschriften, respektive Kinderzeitschriften von einem (ausgewählten) ihm zugedachten Zielpublikum tatsächlich gelesen werden, - welche Inhalte besonders bevorzugt und gerne gelesen werden, - welche Inhalte der jugendliche Leser vernachlässigt oder gar nicht behandelt sieht, aber gerne dargeboten hätte und inwieweit den religiösen Inhalten der Zeitschriften Bedeutung seitens der Leser beigemessen wird. Als Resultate der Befragung sind zu verzeichnen:

- In einer 160 Personen umfassenden Gruppe von 12- bis 13jährigen Schülern kirchlich geleiteter Schulen sind die national verbreiteten katholischen (kirchlichen) Kinderzeitschriften größtenteils bekannt. Dieser hohe Bekanntheitsgrad wird auf die Situationsbedingtheit der befragten Personen als Schüler kirchlich geleiteter Schulen (und somit auf das auf die Gruppe vermutlich zutreffende Merkmal der Zugehörigkeit zum Intensivsegment katholischer Gläubigen) zurüdkgeführt.

- Unter der aus Knaben und Mädchen anteilmäßig gleich zusammengesetzten Gruppe der Befragten ist der Anteil der männlichen Leser von kirchlichen Kinderzeitschriften größer als jener der weiblichen Leser. Mehr als vier Fünftel (82,4 Prozent) der Leser beziehen die von ihnen gelesenen kirchlichen Kinderzeitschriften über die besuchte Schule; für den Kauf kommen bei 60,2 Prozent der Leser die Eltern auf; 26 Prozent erwerben die Zeitschrift selbst. Der verbleibende Rest entfällt auf Personen, die sich die Zeitschriften von Mitschülern ausleihen.

- Die Lesemotive der jugendlichen Leser gründen in der Suche nach Information, Verhaltensorientierung und Unterhaltung in auffallend gleicher Weise. Insofern kommt den kirchlichen Kinderzeitschriften sowohl Informations-, Verhaltensorientierungs- wie auch Soziusfunktion in gleicher Bedeutung zu.

- Alle Leser der kirchlichen Kinderzeitschriften lesen auch andere, meist kommerzielle Jugendmagazine und Illustrierte. Dabei erweist sich der Konsum von Pressemedien, insbesondere kommerzieller Illustrierter, bei Mädchen größer als bei Knaben. Spezifischen Interessen der 12- bis 13jährigen Mädchen wird durch die häufige Nennung von Frauen- und Modezeitschriften Ausdruck verliehen. Die Tatsache des starken Konsums kommerzieler Jugendmagazine, aber auch Illustrierter durch die Leser kirchlicher Kinderzeitschriften stellt die kirchliche Jugendpublizistik vor das Faktum der Konkurrenz mit diesen Zeitschriften.

- Hinsichtlich der Inhalte kirchlicher Kinderzeitschriften kann aus der Analyse der Themengefälligkeit eine positive Beurteilung vor allem des Komplexes der Unterhaltungs- und Freizeitgestaltungsinhalte festgestellt werden. Zugleich wird aber auch das besondere Interesse der Leser an den in den kirchlichen Kinderzeitschriften vernachlässigten Bereichen des ,Nebeneinanders mit dem anderen Geschlecht ${ }^{`}$ (Liebe und Sex) sowie der Einfügung in die gesellschaftliche Umwelt und ihre Bedingungen (Sozialisation und Politik) uiberaus deutlich.

- Die sozialen und caritativen Intentionen kirchlicher Jugendpublizistik erfreuen sich - wenn auch bei einem kleineren Teil der Leser - besonderer Beachtung. Dagegen trifft dies für die religiösen Intentionen nicht zu. 
Interpretation: zum Kommunikationsgefüge kirchlicher Jugendpublizistik in Österreich

Wir haben in der vorliegenden Studie anhand ausgewählter kirchlicher Jugendzeitschriften kirchliche Jugendpublizistik - und somit Phänomene der sozialen Kommunikation der Kirchen - untersucht. Die Ergebnisse führen zu Uberlegungen, die das Kommunikationsgefüge kirchlicher Jugendpublizistik in Österreich wie folgt interpretieren lassen:

Die in der Mehrheit nicht mit kirchlichen Weihen ausgestatteten oder theologisch vorgebildeten Redakteure kirchlicher Jugendzeitschriften fühlen sich nicht in einem weisungsgebundenen Abhängigkeitsverhältnis zur Kirche, identifizieren sich aber mit den Zielen und weitgehend auch mit den Methoden kirchlicher Jugend- und Bildungsarbeit. Sie sehen ihre primären Aufgaben in der Intensivierung der Berichterstattung über religiöse, soziale und sozialkaritative Themen, deren Funktion sie als Handlungsorientierung und Handlungsanweisung artikulieren. Diese Intentionen der Kommunikatoren manifestieren sich in den von ihnen verantworteten und produzierten Zeitschriften hinsichtlich der zeitbezogenen Präsentation speziell religiöser Inhalte nur teilweise. Obwohl nun die Begrenztheit der pastoralen Ansprechbarkeit insbesondere von Jugendlichen bekannt ${ }^{29}$, ihr Interesse an religiösen Fragen jedoch nicht gering ist ${ }^{30}$, wird es um so mehr gelten, religiöse und theologische Fragen als Informationen mit entsprechenden Begründungen und speziell sozialem Bezug darzustellen ${ }^{31}$, um die gesellschaftliche Relevanz der durch Kirche institutionalisierten Religion dem jungen Menschen verständlich zu machen. Die Tatsache, daß die Redakteure kirchlicher Jugendzeitschriften behaupten, sich an einen möglichst breiten, nicht ausschließlich konfessionell gebundenen Leserkreis zu wenden, verleiht diesem Postulat besonderen Nachdruck. Zwar bezeichnen die Redakteure die Leser kirchlicher Jugendpresse als zu passiv-rezipierend, andererseits wollen sie sie nicht (allein) als zu Beeinflussende, sondern auch als Inspiratoren und Gesprächspartner betrachten. Dem kann ein bestimmter funktional-kommunikativer Akzent nicht abgesprochen werden.

Die 12 formal- und inhaltsanalytisch bearbeiteten Pressemedien kirchlicher Jugendpublizistik in Osterreich lassen sich innerhalb einer Typologie als Kinder-, Jugendund Führungszeitschriften zusammenfassen. Der Anteil der Auflage katholischer und evangelischer Zeitschriften verhält sich - fast gemäß der Realpräsenz dieser beiden Kirchen - 94 zu 6 Prozent, der Anteil der Zeitschriftentitel 2 zu 1. Bezüglich ihrer äußeren Erscheinung sind dabei die katholischen Zeitschriften allgemein drucktechnisch aufwendiger gestaltet als die evangelischen. Hinsichtlich ihres inbaltlichen $\mathrm{Ge}$ füges scheinen in den analysierten Zeitschriften religiöse, kirchlich institutionelle, säkulare und Unterhaltungsartikel auf. Dabei stellen die religiösen Artikel die kleinste Inhaltsgruppe in allen Zeitschriften dar. Ihr geringer Anteil (nur 9,9 Prozent) widerlegt die Aussagen eines Großteils der Redakteure, wonach sie religiösen Artikeln besondere Beachtung schenken. Innerhalb der kirchlichen Jugendarbeit läßt die Beridhterstattung der kirchlichen Jugendzeitschriften die spezifische Heilssorge der Kirchen ${ }^{32}$ sowie den Stellenwert religiös-relevanter Bildungsgüter und deren sozialen Sinngehalt nicht deutlich genug erkennen. Als ebenfalls relativ kleine Gruppe erscheinen (mit 22,6 Prozent) die kirchlich-institutionellen Artikel. Diese in den für die praktische Jugendarbeit bedeutungsvollen Führungszeitschriften am häufigsten auftretenden Inhalte erweisen sich als unterschiedlich bei den katholischen bzw. evangelischen Zeitschriften: das kirchliche Informationsgebaren ${ }^{33}$ katholischer Jugendpublizistik bezüglich kirchlicher Binneninformation sowie kirchlicher Information für die 
,jugendliche Offentlichkeit ${ }^{\star}$ erschöpft sich in der Darstellung sozial-caritativer Leistungen und kirchlich institutioneller Veranstaltungen verschiedener Jugendorganisa. tionen. Dagegen nehmen die Artikel der evangelischen Zeitschriften sowohl zu innerkirchlichen Vorgängen Stellung, zeigen das Verhältnis des Evangelischen Jugendwerkes zur Gesamtkirche auf und versuchen weiters, eine permanente Darstellung von Selbstverständnis und Ziel evangelischer Jugendarbeit zu geben - ein Informationsgebaren, das Binnen- und Offentlichkeitsinformation zugleich gewährleistet und dem Postulat der Pflicht zur Information im gegenseitigen Informationsaustausch zwischen Kirche und Offentlichkeit gerecht $\mathrm{zu}$ werden versucht, zugleich aber auch (unumschränkte) Transparenz über innerkirchliche Vorgänge bietet und „kritisches, selbstkritisches Bewußtsein auch in der Kirche" erreichen will. ${ }^{34}$

Einen überraschend hohen Anteil verzeichnet als größte Inhaltsgruppe die Unterhaltungsberichterstattung, die in den Kinderzeitschriften am häufigsten auffindbar ist und vor allem in drei auflagenstarken katholischen Blättern durch ihre Artikel über den gesamten Freizeitbereich, Unterhaltungsmusik, Film, Fernsehen, Stars und Idole sowohl in Inhalt wie auch in Ausstattung deutliche Anpassungstendenzen an die kommerzielle Jugendpresse zeitigt. Das Wissen um die Konkurrenz kirchlicher Jugendpublizistik mit dem System der Freizeitgestaltung ${ }^{35}$ erscheint somit erwiesen.

Als verhältnismäßig universell und, soweit rekonstruier- und überprüfbar, aktuell präsentiert sich die vor allem in den Jugendzeitschriften auffallend häufig vorfindbare säkulare Berichterstattung, die ihr inhaltliches Spektrum über Politik, Zeitgeschichte, Dritte Welt, insbesondere aber über Technik, Natur, Soziales, Kultur, Sport, Schule, Beruf und Erziehung spannt. Sie bestätigt nachweislich die von den Redakteuren besonders betonten sozialen und sozial-karitativen Ziele, vernachlässigt aber die in Programmen kirchlicher Jugendarbeit ${ }^{36}$ aufscheinenden Absichten der ,Lebenshilfe ${ }^{c}$ vor allem in den Bereichen des Partnerproblems, der Bewältigung des beruflichen Alltags sowie der Lebensorientierung in Staat und Gesellschaft; ebenso bleiben die für das Verstehen und Funktionieren demokratisch organisierter Sozialgebilde wichtigen Bereiche der politischen Bildung und zurecht geforderten Medienerziehung" unberïcksichtigt.

In einer ausgewählten Gruppe von Lesern (Kindern) kirchlicher Kinderzeitschriften gründen die Lesemotive in der Suche nach Information, Unterhaltung und Verhaltensweisen: ein Resultat, das der Funktion zumindest kirchlicher Kinderzeitschriften im Sinne der Verhaltens- und Handlungsorientierung Ausdruck verleihen soll, zumal wir auch beweisen können, daß der Konsum kommerzieller Jugendmagazine und Illustrierten durch den befragten Personenkreis beachtlich ist. Dieses Faktum stellt die kirchliche Jugendpublizistik vor das notgedrungen zu fordernde Bemühen, mit ihren Zeitschriften eine adäquate Alternative zu den kommerziellen Magazinen darzustellen; dies auch deshalb, weil ein besonderes Interesse bereits bei den Kindern an den in den kirchlichen Jugendzeitschriften tabuisierten Bereichen des Nebeneinanders mit dem anderen Geschlecht wie auch der gesellschaftlichen Sozialisation (Einfügung in die gesellschaftliche Umwelt und ihre Bedingungen) feststellbar ist.

Kirchliche Pressejugendpublizistik in Osterreich erweist sich unseren Untersuchungsergebnissen zufolge nicht als Beitrag zur ,Verkündigung im Zeitalter der Massenmedien', sondern als Ubbermittler(in) relativ breit gestreuter, den Interessen junger Menschen angeglichener Informationen. Die Analyse kann den in der publizistikwissenschaftlichen Fachliteratur vorfindbaren, kirchlicher Publizistik allgemein zugeschriebenen und kritisierten normativen, vertikal von oben nach unten verlaufenden 
Kommunikationsfluß nicht (oder doch nicht unbedingt) bestätigen, wohl aber funktionale Elemente zwischen Kommunikator und Rezipient (ansatzweise) feststellen. Dennoch wird hier an die kirchliche Jugendpublizistik OOsterreichs die Forderung gestellt, sich nicht nur in der Konkurrenz mit der kommerziellen Jugend- und Publikumspresse an Informationen und deren formaler Präsentation zu messen, sondern vielmehr durch bewußte Kenntnisnahme und Akzeptieren der Anliegen und Wünsche junger Menschen ${ }^{38}$ sich an nüchterner und sachgebundener Lebenshilfe ${ }^{50}$ in Konfliktbereichen der Lebens- und Glaubensfragen junger Menschen zu orientieren, um damit einen sozialrelevanten Beitrag zur gesellschaftlichen Kommunikation zu leisten.

\section{Anmerkungen:}

1. Vgl. Franz Kardinal König: Kirche und Kommunikation. In: CS 1:1968, S. 6-20; vgl. Siegfried von Kortzfleisch: Verkündigung und öffentliche Meinungsbildung. Ein Beitrag zur Grundlegung kirchlicher Offentlichkeitsarbeit. Stuttgart: Evangelisches Verlagswerk 1960, S. 116 ff.

2. Paul Hastenteufl: Jugendbewegung und Jugendseelsorge. Geschichte und Probleme der katholischen Jugendarbeit im 20. Jahrhundert. München: Kösel 1962, S. 11.

3. Vgl. Hastenteufl, a.a.O. S. 11 ff.; Hastenteufl beschreibt und erklärt die Entwicklung der kirchlichen (katholischen) Jugendarbeit in Deutschland und Osterreich. Darauf im besonderen einzugehen ist nicht Aufgabe der vorliegenden Untersuchung.

4. Für Osterreich ist damit publizistikwissenschaftliches Neuland betreten, zumal zumindest auf dem Sektor der kirchlichen Publizistik nur einige wenige weiterführende Arbeiten bekannt sind, die außerdem immer nur eine Position des publizistischen Prozesses untersuchen. Vgl. Julius Morel: Seelsorge im Spiegel der Presse. Ergebnisse einer Inhaltsanalyse. In: „Diakonia - Der Seelsorger“. Internationale Zeitschrift für praktische Theologie. 1:1970, S. 200-205. Henrik Kreutz: Einfluß von Massenmedien, persönlicher Kontakt und formelle Organisation. Kritik und Weiterführung der These ,two step flow of communication'. In: Franz Ronneberger (Hrsg.): Sozialisation durch Massenmedien. Der Mensch als soziales und personales Wesen. 4. Band. Stuttgart: Enke 1971, S. 173-241. Karl Bauer-Debois: Pädagogisch-psychologisches Fachgutachten von österreichischen Kinderzeitschriften. Unveröff. Manuskript, Wien 1968.

5. „Unter Strukturuntersuchung wird verstanden die Ermittlung von nachprüfbaren Daten über das äußere und innere Gefüge" (von Zeitungen oder Zeitschriften). Vgl. Günter Kieslich: Die Struktur der österreichischen Tagespresse 1969. Ergebnisse einer Untersuchung des Instituts für Publizistik und Kommunikationstheorie der Universität Salzburg. Salzburg 1970 („arbeitsberichte 1“), S. 8.

6. Die für eine solche Untersuchung notwendig zu treffenden Auswahlen werden in den einzelnen Abschnitten behandelt.

7. Vgl. dazu Manfred Rühl: Systemdenken und Publizistikwissenschaft. In: „Publizistik“ 14:1969, S. $185 \mathrm{ff}$; vgl. ebenso Franz Dröge: Theorie und Erkenntnistheorie in der Publizistikwissenschaft. In: „Publizistik“ $12: 1967$, S. 219 ff.; vgl. Joachim Westerbarkey: Zur Kommunikationssoziologie des Katholizismus. In: CS 4:1971, S. 209-225; vg1. Hans Magnus Enzensberger: Baukasten zu einer Theorie der Medien. In: „Kursbuch 20“, S. $173 \mathrm{ff}$.

8. Ein normatives Verständnis von Publizistik wurde z. B. von Emil Dovifat artikuliert; vgl. Emil Dovifat (Hrsg.): Handbuch der Publizistik, Bd. 1, Berlin: de Gruyter 1968, S. 5.

9. Michael Schmolke: Fragen zum Ort von Gesinnungskräften im publizistischen Funktionieren. In: CS $1: 1968$, S. 109.

10. Schmolke, a.a.O. S. 109.

11. Schmolke, a.a.O. S. 109. 
12. Vgl. Westerbarkey, a.a.O. S. $212 ; \ldots$... Die hierarchische Organisationsstruktur (Herrschaftsstruktur) des katholischen Klerus ist durch den Primat formal abwärtsgerichteter Kommunikation gekennzeichnet, also durch vertikale Kanäle der Instruktion, Koordination und Kontrolle. Je straffer eine Hierarchie organisiert ist, desto schwächer scheint der Fluß aufwärtsgerichteter Kommunikation zu fließen. In der hierarchischen Struktur des Klerus dienen Untergebene primär zur Rezeption und Ausführung ..." (S. 212). Der Informationsfluß von unten nach oben hingegen erfolgt in der Form des Berichtes, schließt aber zumeist jegliche Form der Kritik aus.

13. Vgl. Franz W. Dröge: Publizistik und Vorurteil. Münster: Regensburg 1967. („Dialog der Gesellschaft 4"), S. 37.

14. Vgl. Henk Prakke und Gerhard E. Stoll: Entwurf zu einer Religionspublizistik. In: CS $1: 1968$, S. 35; siehe auch Gerhard E. Stoll: Erwägungen zur religiösen Publizistik. In: "Publizistik" 10:1965, S. 15-21.

15. Hier ist unter anderem die Problematik der "Verkündigung" durch Massenmedien angesprochen. Protestantische und katholische Lehrtradition unterscheiden sich hinsichtlich der Basis der Verkündigung in der unterschiedlichen Wertung von Schrift und Tradition. Die katholische Lehre fundiert sowohl auf Schrift als auch auf Tradition, wobei Tradition hier durch die amtlich abgesicherte Interpretation der Schriften durch die Kirchenväter wie auch durch die Gesamtkirche meint. Die protestantische Kirche dagegen verkündigt nach dem "sola Scriptura"-Prinzip. Vgl. Edward Schillebeeckx: Offenbarung und Theologie. Mainz: Grünewald 1965, S. $26 \mathrm{f}$. Auf die in der Fachliteratur stark diskutierte Verkündigungsproblematik und auf die dabei aufscheinenden unterschiedlichen Verständnisse von Verkündigung und Verkündigung durch Massenmedien kann hier nicht eingegangen werden (vgl. in der Dissertation die Seiten 18-24).

16. Vgl. Gerhard Maletzke: Psyochologie der Massenkommunikation. Theorie und Systematik. - Hamburg: Bredow 1963, S. 77.

17. Schmolke, a.a.O. S. 109.

18. Vgl. Thomas A. Bauer: Theorien religiöser Kommunikation. Seminararbeit am Institut für Publizistik und Kommunikationstheorie der Universität Salzburg. Unveröffentl. Manuskript. Salzburg 1971, S. 15. Vgl. auch Maletzke, a.a.O. S. $77 \mathrm{ff}$.

19. Im Folgenden wird, so nicht ausdrücklich getrennt, für Kinder- und Jugendzeitschriften der Sammelbegriff, Jugendzeitschriften' verwendet.

20. Vgl. Bernhard Klaus: Massenmedien im Dienst der Kirche. Theologie und Praxis. Berlin: de Gruyter 1969, S. 20.

21. Vgl. Kieslich, a.a.O. S. 8.

22. Vgl. Elisabeth Noelle-Neumann und Winfried B. Schulz (Hrsg.): Publizistik. Frankfurt/ Main: Fischer 1971 (= Fischer Lexikon 9), S. 51.

23. Ab hier wieder Sammelbegriff.

24. Vgl. Erika Weinzierl: Die katholische Kirche. In: Erika Weinzierl und Kurt Skalnik (Hrsg.): Osterreich. Die zweite Republik, 2. Bd. Graz-Wien-Köln: Styria 1972, S. 285319.

25. Großgruppe, religiöse Artikel‘: Theologie, Spiritualität, Verkündigung, Gemeinde, Leitbilder; Großgruppe ,Kirchlich-institutionelle Artikel': Kirche allgemein, Hierarchie, Priester, kirchliche Gliederungen, Sozial-Karitatives, Kirchlich-Kulturelles; Großgruppe ,Säkulare Artikel': Politik, Soziales, Kultur/Reise, Sport, Zeitgeschichte, Wissenschaft/Technik, Dritte Welt, Schule/Beruf/Erziehung, Sonstiges; Großgruppe ,Unterhaltungsartikel': Film/Fernsehen, Mode, Schlager/Pop/Folk, Basteleien/Kochen, Rätsel, Freizeit allgemein, Romane/Erzählungen. Ihre genaue Definition ist in der diesem Aufsatz zugrundeliegenden Dissertation erfolgt.

26. Der Erhebungsbogen beinhaltete nicht nur thematisch-inhaltliche Kategorien, sondern auch publizistisch formale Merkmale, die erfaßt werden sollten: die publizistische Form (informative, interpretative, kommentierende, meditative Artikel), Länge der Artikel und Illustrierungsgrad.

27. Für ,Artikel' noch synonym gebraucht: Aussageeinheit, Text.

28. Vgl. Tabelle 2 auf Seite 144.

29. Vgl. Siegfried Baumgartner: Jugendzeitschriften in der Krise. In: CS 1:1968, S. 244. 
30. Vgl. Paul Jakobi: Jugend und Kirche. In: Dieter Stoll (Hrsg.): Kirche in der Diskussion. Umfragen, Kommentare, Analysen zur Zukunft der Kirche. Düsseldorf, Altenberg 1970, S. 61; Franz Huemer: Jugend heute in der Sicht des Religionspädagogen. In: Gefährdete Jugend. Was können wir tun, daß sie ihre Chance nützt. Wien-Linz-Passau: Veritas 1971; Wolfgang Marhold: Fragende Kirche. Über Methode und Funktion kirchlicher Meinungsumfragen. München: Kaiser, Mainz: Grünewald 1971, S. 105-107.

31. Vgl. Jakobi, a.a.O. S. 61.

32. Vgl. Paul Hastenteufl: Die Bildungsgüter der kirchlichen Jugendarbeit. In: "Jugend und Kirche $^{\alpha} 3: 1970$, Heft 5, S. 4 f.

33. Vgl. Michael Schmolke: Kirchenpresse und innerkirchliche Information. In: CS 5:1972, S. 126.

34. Johannes Dantine: Der "anstoss" - Abenteuer einer evangelischen Jugendzeitschrift. In: "Theologia practica" 1971 , S. 60.

35. Vgl. Klaus, a.a.O. S. $66 \mathrm{f}$.

36. Vgl. Hastenteufl: Bildungsgüter, a.a.O. S. 10.

37. Nicht parteipolitische Bildung; zu Medienerziehung siehe: Franz Zöchbauer: Medienerziehung. In: Gefährdete Jugend, a.a.O. S. 30-33; denselben, Jugend und Fernsehinformation. In: CS 1:1968, S. 225-231; denselben, Verkündigung im Zeitalter der Massenmedien. München: Kösel 1969, S. 19-26.

38. Vgl. Baumgartner, a.a.O. S. 248.

39. Vgl. H. Boventer: Moderne Jugendpresse. Aus einem Referat. Unveröff. Manuskript 1964, S. 20.

\section{S U M M A R Y}

The author analyses the communicative structure of the christian youth press in Austria. Using empirical methods, editors, media contents and readers are analysed in order to get a picture of the catholic and protestant press for and of young people. The analysis reveals that church publications for this age are not primarily contributing to evangelization. They are more devoted to relatively widespread information according to the interest of children and young people. A normative vertical flow from top to bottom as usually attributed to church communications is not to be found in the analysed publications. Communicator and recipient are at least beginning to be equal partners. The catholic publications are very much adjusted to youth publication of general interest.

\section{RÉSUME}

Dans l'étude présente, on analyse la structure de communication de la publicistique religieuse pour la jeunesse, en Autriche. On examina à l'aide de méthodes d'analyse empiriques les rédacteurs, moyens, contenus et lecteurs de magazines pour enfants et adolescents, publiés a l'échelon national, pour parvenir à des déclarations au sujet de la structure de communication de la publicistique religieuse (catholique et évangélique) pour la jeunesse. La presse religieuse pour la jeunesse s'est montrée à cette occasion non pas primaire en tant que contribuant à la propagation à l'époque des moyens de masse, mais servant à retransmettre sur un relativement vaste éventail des informations ajustées aux centres d'intérêts des enfants et des adolescents. Un courant de communication religieux, dédié en commun à la communication et à la publicistique, normatif et circulant verticalement de "haut" en "bas", ne peut être constaté en matière de publicistique religieuse pour la jeunesse. Dans les débuts au moins, on peut reconnaître communicateurs et receveurs comme partenaires. Les revues (catholiques) alles-mêmes, sont assez fortement adaptées au style de la presse (non religieuse) pour la jeunesse, en général. 